\title{
DISCONTINUOUS TRANSLATION INVARIANT FUNCTIONALS
}

BY

SADAHIRO SAEKI

Dedicated to Professor Shigeki Yano on his sixtieth birthday

\begin{abstract}
Let $G$ be an infinite $\sigma$-compact locally compact group. We shall study the existence of many discontinuous translation invariant linear functionals on a variety of translation invariant Fréchet spaces of Radon measures on $G$. These spaces include the convolution measure algebra $M(G)$, the Lebesgue spaces $L^{p}(G)$, where $1 \leqslant p \leqslant \infty$, and certain combinations thereof. Among other things, it will be shown that $C(G)$ has many discontinuous translation invariant functionals, provided that $G$ is amenable. This solves a problem of G. H. Meisters.
\end{abstract}

Let $G$ be a locally compact group. In the present paper, we shall consider various translation invariant spaces of functions and measures on $G$, and prove the existence of discontinuous invariant functionals on such spaces. Among other things, it will be shown that $C(G)$ admits "many" discontinuous left-invariant functionals, provided that $G$ is an infinite, $\sigma$-compact, locally compact amenable group (our hypotheses are a little weaker than amenability). This resolves the problem of G. H. Meisters [11] as to whether there exists a discontinuous invariant functional on $C(\Pi)$, where $\Pi$ denotes the circle group. As far as the compact groups are concerned, the problems for $C(G)$ and for $L^{\infty}(G)$ are equivalent (see Remark (IV) stated below). As some basic references on this subject, we refer to E. Granirer's papers [3,4] on invariant means, the nice survey article [14] by Meisters, and G. S. Woodward's paper [22] on discontinuous invariant functionals on a variety of invariant spaces.

Let $G$ be an arbitrary locally compact (LC) group. All of the Lebesgue spaces $L^{p}(G), 1 \leqslant p \leqslant \infty$, are taken with respect to a fixed left Haar measure $\lambda_{G}$ on $G$. If $G$ is compact, we normalize $\lambda_{G}$ so that $\lambda_{G}(G)=1$. Let $C(G)$ and $C_{c}(G)$ denote the Banach space of all continuous bounded (complex-valued) functions on $G$, and the subspace of all members of $C(G)$ with compact supports, respectively. We denote by $C_{0}(G)$ the uniform closure of $C_{c}(G)$ in $C(G)$. Thus the dual of $C_{0}(G)$ may be identified with the convolution measure algebra $M(G)$ of $G$ (cf. Hewitt and Ross [7]).

A net $\left(f_{\alpha}\right)$ in $C_{c}(G)$ converges to the zero function if and only if (i) $f_{\alpha} \rightarrow 0$ uniformly on $G$ and (ii) the $f_{\alpha}$ 's are eventually supported by a fixed compact set (depending on the net). This topology for $C_{c}(G)$ is different from the uniform topology, and will be used throughout the paper unless otherwise mentioned. The continuous dual of $C_{c}(G)$ will be denoted $C_{c}^{\prime}(G)$. An element of $C_{c}^{\prime}(G)$ is often called

Received by the editors February 8, 1983 and, in revised form, April 19, 1983.

1980 Mathematics Subject Classification. Primary 43A15; Secondary 43A05.

Key words and phrases. Translation invariant Fréchet space, discontinuous invariant functional, Radon measure. 
a Radon measure on $G$ (cf. [16]). If a linear subspace $X$ of $C_{c}^{\prime}(G)$ is equipped with a topological-linear-space topology which is stronger than the weak topology $\sigma\left(X, C_{c}(G)\right)$, we call $X$ a topological linear space on $G$. Normed, Banach, and Fréchet spaces on $G$ are defined in a similar fashion. Notice that if $X$ and $Y$ are two Fréchet spaces on $G$ such that $X \subset Y$, then the identity map of $X$ into $Y$ is continuous by the closed graph theorem. We shall regard all of the spaces $L^{p}(G) \subset L_{\mathrm{loc}}^{1}(G)$ and $M(G)$ as linear subspaces of $C_{c}^{\prime}(G)$. In particular, if $f \in L_{\mathrm{loc}}^{1}(G)$, then

$$
\langle\phi, f\rangle=\left\langle\phi, f \lambda_{G}\right\rangle=\int \phi(x) f(x) d \lambda_{G}(x) \quad\left(\phi \in C_{c}(G)\right) .
$$

Similarly, $M(G)+L^{p}(G)$ denotes the algebraic sum of $M(G)$ and $L^{p}(G)$ in $C_{c}^{\prime}(G)$.

For each $\phi \in C_{c}(G)$ and $a \in G$, define ${ }_{a} \phi$ and $\phi_{a} \in C_{c}(G)$ by setting ${ }_{a} \phi(x)=\phi(a x)$ and $\phi_{a}(x)=\phi(x a)$ for all $x \in G$. Let $\delta_{a}$ denote the unit point-measure at $a$. Thus we have

$$
\left\langle\phi, \delta_{a} * \mu\right\rangle=\left\langle{ }_{a} \phi, \mu\right\rangle \text { and }\left\langle\phi, \mu * \delta_{a}\right\rangle=\left\langle\phi_{a}, \mu\right\rangle
$$

for $\phi \in C_{c}(G)$ and $\mu \in C_{c}^{\prime}(G)$. Here follow some other definitions which we shall need later.

(a) A subset $X$ of $C_{c}^{\prime}(G)$ is left invariation if $\delta_{a} * X \subset X$ for all $a \in G$. Right- (or two-sided) invariance is defined similarly.

(b) For a subset $X$ of $C_{c}^{\prime}(G)$, we define

$T_{l}(X)=\operatorname{sp}\left\{f-\delta_{a} * f: f \in X \& a \in G\right\}, \quad T_{r}(X)=\operatorname{sp}\left\{f-f * \delta_{a}: f \in X \& a \in G\right\}$, and $T(X)=T_{l}(X)+T_{r}(X)$, where "sp" stands for linear span.

(c) If $X$ and $Y$ are two topological spaces such that $X \subset Y$ set-theoretically, we shall write " -" for the closure in the smaller space $X$ and " $~$ " for the closure in the larger space $Y$.

(d) For a topological linear space $X, X^{\prime}$ denotes the continuous dual of $X$. If $X$ is a normed space and $r>0$, we define $B_{r}(X)=\{f \in X:\|f\| \leqslant r\}$.

In the sequel, $G$ will always denote an infinite $\sigma$-compact LC group, unless otherwise mentioned. The following two lemmas are central to all of our results. Their natural "two-sided" versions are obvious and will be left to the reader.

Lemma 1. Let $X$ and $Y$ be a left-invariant Fréchet space and a left-invariant Banach space on $G$, respectively. Suppose that

(i) $X \subset Y$ and $T_{l}(X)^{-} \subset \mathrm{sp}(F)+T_{l}(Y)$

for some countable subset $F$ of $Y$. Then there exist a natural number $N$ and a compact subset $K$ of $G$ such that $W_{N, K}^{\sim}$ contains a relative neighborhood of 0 in $T_{l}(X)^{-}$(with respect to $X$ ), where

(ii) $W_{N, K}=\left\{\sum_{k=1}^{N}\left(h_{k}-\delta_{a_{k}} * h_{k}\right): h_{k} \in B_{N}(Y) \& a_{k} \in K \forall k\right\}$.

Proof. Let $f_{1}, f_{2}, \ldots$ be an enumeration of the elements of $F$. Since $G$ is $\sigma$-compact, we can write $G=\cup_{n=1}^{\infty} K_{n}$, where the $K_{n}$ 's are compact subsets of $G$ with $K_{n} \subset K_{n+1}$ for all $n \geqslant 1$. Given a natural number $n$, define

$$
V_{n}=\left\{\sum_{j=1}^{n} c_{j} f_{j}: c_{j} \in \mathbf{C} \&\left|c_{j}\right| \leqslant n \forall_{j}\right\} .
$$


Likewise, define $W_{n}$ by (ii) with $N$ and $K$ replaced by $n$ and $K_{n}$, respectively. Notice that $V_{n}$ is compact. Since the algebraic sum of a compact set and a closed set is closed in any topological linear space, it follows that $V_{n}+W_{n}^{\sim}$ is closed in $Y$.

By (i), the Fréchet space $T_{l}(X)^{-}$is covered by the sets $V_{n}+W_{n}^{\sim}$. Since $T_{l}(X)^{-}$is contained in the Banach space $Y$ on $G$, it follows from the closed graph theorem that each $\left(V_{n}+W_{n}^{\sim}\right) \cap T_{l}(X)^{-}$is closed in $T_{l}(X)^{-}$. We, therefore, infer from the Baire category theorem that at least one of the sets $V_{n}+W_{n}^{\sim}$ contains a nonempty (relative) open subset of $T_{l}(X)^{-}$. However, we have $V_{n}-V_{n} \subset V_{2 n}$ and $m V_{n} \subset V_{m n}$ for all $m$ and $n \geqslant 1$ by (1); similarly for the sets $W_{n}^{\sim}$. Accordingly, there exists a natural number $r$ such that $V_{r}+W_{r}^{\sim}$ contains a neighborhood $U$ of $0 \in T_{l}(X)^{-}$.

Now we claim that $U \subset W_{N}^{\sim}$ for some $N \geqslant r$. To confirm this, first notice that the union of all $W_{n}^{\sim}$ forms a linear subspace of $Y$. Call this union $S$ and put $F_{p}=\left\{f_{1}, \ldots, f_{p}\right\}$ for $p=1,2, \ldots, r$. If $F_{r} \subset S$, then $F_{r} \subset W_{m}^{\sim}$ for some $m$; hence $V_{r} \subset W_{n}^{\sim}$ for some $n>m$ by (1) and (ii); hence $V_{r}+W_{r}^{\sim} \subset W_{n}^{\sim}+W_{r}^{\sim} \subset W_{N}^{\sim}$, where $N=n+r$. Thus $F_{r} \subset S$ implies $U \subset W_{N}^{\sim}$ for some $N$. So assume that $F_{r}$ is not contained in $S$. Then we may suppose that, for some $p \leqslant r, F_{p}$ is a maximal subset of $F_{r}$ which is linearly independent modulo $S$. After repeating a similar argument as above, we then obtain

$$
U \subset V_{r}+W_{r}^{\sim} \subset \operatorname{sp}\left(F_{p}\right)+W_{N}^{\sim}
$$

for some $N \geqslant r$. Notice that $T_{l}(X) \subset T_{l}(Y) \subset S$; hence

$$
T_{l}(X) \cap\left[\operatorname{sp}\left(F_{p}\right)+W_{N}^{\sim}\right]=T_{l}(X) \cap W_{N}^{\sim}
$$

by the linear independence of $F_{p}$ modulo $S$. We infer from (2) and (3) that $U \cap T_{l}(X) \subset W_{N}^{\sim}$. Since $\left[T_{l}(X)^{-}\right] \cap W_{N}^{\sim}$ is closed in $T_{l}(X)^{-}$, it follows that $U \subset$ $W_{N}^{\sim}$, as desired.

Lemma 2. Let $X$ be a left-invariant Fréchet space on $G$, and let $Y$ be a left-invariant Banach space on $G$ such that

$\left(\mathrm{C}_{0}\right) X \subset Y$ and $T_{l}(X)^{-} \subset \mathrm{sp}(F)+T_{l}(Y)$

for some countable subset $F$ of $Y$. Suppose

$\left(\mathrm{C}_{1}\right)$ the unit closed ball $B_{1}(Y)$ is $\sigma\left(Y, C_{c}\right)$-compact;

$\left(\mathrm{C}_{2}\right)$ the orbit $\left\{\delta_{x} * f: x \in G\right\}$ of each $f \in X$ is bounded in $X$;

and also (if $G$ is noncompact)

$\left(\mathrm{C}_{3}\right)$ for which $f \in X, \lim _{x \rightarrow \infty} \delta_{x} * f=0$ in $\sigma\left(X, C_{c}\right)$.

Let $W_{N, K} \subset Y$ be as in the conclusion of Lemma 1. Then:

(i) $W_{N, K}$ is $\sigma\left(Y, C_{c}\right)$-compact;

(ii) if $G$ is noncompact, $W_{2 N, K}$ contains a neighborhood of $0 \in X$;

(iii) if $G$ is compact, there exists a neighborhood $V$ of $0 \in X$ such that $h-\lambda_{G} * h \in$ $W_{2 N, K}$ for all $h \in V$.

PRoOF. For each open subset $U$ of $G$ with compact closure, let $C_{U}$ denote the subspace of all $\phi \in C_{c}(G)$ such that supp $\phi \subset U^{-}$. Then $C_{U}$ forms a Banach space with respect to the uniform norm and the imbedding of $C_{U}$ into $C_{c}(G)$ is continuous. Since $Y$ is a Banach space contained in $C_{c}^{\prime}(G)$, the closed graph theorem assures that 
there exists a finite constant $D=D_{U}$ such that

$$
|\langle\phi, h\rangle| \leqslant D\|\phi\|_{\infty} \cdot\|h\|_{Y} \quad\left(\phi \in C_{U}, h \in Y\right) .
$$

Now we claim that the mapping

$$
(x, h) \rightarrow \delta_{x} * h: G \times B_{1}(Y) \rightarrow C_{c}^{\prime}(G)
$$

is continuous when $B_{1}(Y)$ is equipped with the relative weak topology $\sigma\left(Y, C_{c}\right)$ | $B_{1}(Y)$. Suppose that $\left(x_{\alpha}, h_{\alpha}\right)$ is a convergent net in $G \times B_{1}(Y)$ with limit $(x, h)$. We need to prove that $\lim \delta_{x_{\alpha}} * h_{\alpha}=\delta_{x} * h$ in $\sigma\left(C_{c}^{\prime}, C_{c}\right)$. Given $\phi \in C_{c}(G)$, choose an open subset $U$ of $G$ such that $U^{-}$is compact and $\operatorname{supp}\left({ }_{x} \phi\right) \subset U$. Since $x_{\alpha} \rightarrow x$ in $G$, it is obvious that ${ }_{x_{\alpha}} \phi \in C_{U}$ for eventually all $\alpha$ 's. It follows from (4) that

$$
\begin{aligned}
\left|\left\langle\phi, \delta_{x_{\alpha}} * h_{\alpha}\right\rangle-\left\langle\phi, \delta_{x} * h\right\rangle\right| & =\left|\left\langle_{x_{\alpha}} \phi, h_{\alpha}\right\rangle-\left\langle{ }_{x} \phi, h\right\rangle\right| \\
& \leqslant\left|\left\langle_{x_{\alpha}} \phi-{ }_{x} \phi, h_{\alpha}\right\rangle\right|+\left|\left\langle_{x} \phi, h_{\alpha}-h\right\rangle\right| \\
& \leqslant D\left\|_{x_{\alpha}} \phi-{ }_{x} \phi\right\|_{\infty}+\left|\left\langle{ }_{x} \phi, h_{\alpha}-h\right\rangle\right|
\end{aligned}
$$

for eventually all $\alpha$ 's. Since ${ }_{x_{\alpha}} \phi \rightarrow{ }_{x} \phi$ uniformly and $h_{\alpha} \rightarrow h$ in $\sigma\left(Y, C_{c}\right)$, the last inequalities confirm our claim.

Put $E_{K}=\left\{h-\delta_{x} * h: h \in B_{1}(Y) \& x \in K\right\}$. From condition $\left(\mathrm{C}_{1}\right)$ and the claim just established, we infer that $E_{K}$ is $\sigma\left(Y, C_{c}\right)$-compact whenever $K$ is a compact subset of $G$. The set $W_{N, K}$ in the conclusion of Lemma 1 is the $N$-fold algebraic sum of $N E_{K}$, and is therefore $\sigma\left(Y, C_{c}\right)$-compact. This confirms (i).

Now we shall prove (ii) and (iii) assuming that

$$
W_{N, K}=\left[Y+\operatorname{sp}\left(\lambda_{G}\right)\right] \cap W_{N, K}^{*},
$$

where $W_{N, K}^{*}$ denotes the $\sigma\left(C_{c}^{\prime}, C_{c}\right)$-closure of $W_{N . K}$ in $C_{c}^{\prime}(G)$. Of course, $\left(\mathrm{C}_{1}\right)^{*}$ is weaker than $\left(\mathrm{C}_{1}\right)$ but is strong enough to guarantee the norm-closedness of $W_{N . K}$ in $Y$. Now choose and fix a convex neighborhood $V$ of $0 \in X$ such that $(V-V) \cap$ $T_{l}(X)^{-} \subset W_{N . K}$. Each $f \in X$ has bounded orbit in $X$ by $\left(\mathrm{C}_{2}\right)$. Accordingly there exists a natural number $n=n_{f}$ such that $n^{-1}\left(\delta_{x} * f\right) \in V$ for all $x \in G$. Hence

$$
n^{-1}\left(f-\delta_{x} * f\right) \in(V-V) \cap T_{l}(X) \subset W_{N, K} \quad(x \in G) .
$$

Suppose $G$ is noncompact. Then $\left(\mathrm{C}_{3}\right)$, combined with (6) and $\left(\mathrm{C}_{1}\right)^{*}$, yields $n^{-1} f \in$ $W_{N, K}$. Since $f \in X$ is arbitrary, it follows that $X \subset \cup_{n=1}^{\infty} n W_{N, K}$. But $W_{N, K}$ is norm-closed in $Y$ by $\left(\mathrm{C}_{1}\right)^{*}$, and $X$ is a Fréchet space contained in the Banach space $Y$; hence $X \cap W_{N, K}$ is closed in $X$ (cf. the proof of Lemma 1). It follows from the Baire category theorem that $X \cap W_{N, K}$ has nonempty interior in $X$. Since $W_{N . K}-$ $W_{N, K} \subset W_{2 N, K}$, we conclude that $W_{2 N, K}$ contains a neighborhood of 0 in $X$, which established (ii).

Finally assume $G$ is compact. Then $C_{c}(G)=C(G), C_{c}^{\prime}(G)=M(G), \sigma\left(C_{c}^{\prime}, C_{c}\right)$ is nothing but the weak-* topology of $M(G)$. Choose a net $\left(\tau_{\alpha}\right)$ of probability measures on $G$, each with finite support, such that $\tau_{\alpha} \rightarrow \lambda_{G}$ weak-*. Then $n^{-1}\left(f-\tau_{\alpha} * f\right) \in$ $W_{N, K}$ for each $\alpha$ by (6) and the convexity of $V$. Passing to the weak-* limit and making use of $\left(\mathrm{C}_{1}\right)^{*}$, we obtain $n^{-1}\left(f-\lambda_{G} * f\right) \in W_{N, K}$. Notice that $\lambda_{G} * X \subset$ $\lambda_{G} * M(G)=\operatorname{sp}\left(\lambda_{G}\right)$. Since $f \in X$ is arbitrary, it follows at once that

$$
X \subset \bigcup_{n=1}^{\infty} n\left[\left\{c \lambda_{G}:|c| \leqslant 1\right\}+W_{N, K}\right] .
$$


Using $\left(\mathrm{C}_{1}\right)^{*}$ and $(7)$, we repeat a similar argument as in the last paragraph to conclude that $\operatorname{sp}\left(\lambda_{G}\right)+W_{2 N, K}$ contains a neighborhood $V^{\prime}$ of $0 \in X$. Thus each $h \in V^{\prime}$ has a representation of the form $h=c \lambda_{G}+\mu$ for some $c \in \mathbf{C}$ and some $\mu \in W_{2 N, K}$. But it is obvious that $\langle 1, \nu\rangle=0$ for all $\nu \in W_{2 N, K} \subset T_{l}(M(G))$. Therefore $\langle 1, h\rangle=c\left\langle 1, \lambda_{G}\right\rangle+\langle 1, \mu\rangle=c$, and

$$
h-\lambda_{G} * h=h-\langle 1, h\rangle \lambda_{G}=\mu \in W_{2 N, K},
$$

as desired.

We abbreviate left- [two-sided] invariant linear functional as LILF [TILF]. It is evident that Lemma 2 as well as Lemma 1 has a natural "two-sided" analogue. All that follows are corollaries to Lemma 2.

THEOREM 1. There exist uncountably many TILF's on $M(G)$ whose restrictions to one of $M_{a}(G)$ or $M_{d}(G)$ are linearly independent modulo the continuous functionals on $M_{a}(G)$ or $M_{d}(G)$.

Proof. The symbols $M_{a}(G)$ and $M_{d}(G)$ denote the absolutely continuous and discrete measures on $G$, respectively. Notice that $M_{a}(G)$ is a closed two-sided ideal while $M_{d}(G)$ is a closed two-sided invariant subalgebra of $M(G)$.

By Zorn's lemma, $T\left(M_{a}(G)\right)^{-}$contains a subset $F_{a}$ which is maximal with respect to the linear independence modulo $T\left(M_{a}(G)\right)$. Similarly there exists a subset $F_{d}$ of $T\left(M_{d}(G)\right)^{-}$which is maximal with respect to the linear independence modulo $T\left(M_{d}(G)\right)$. If $G$ is discrete, then $M_{a}(G)=M_{d}(G)=M(G)$, so we shall choose $F_{a}=F_{d}$. If $G$ is nondiscrete, it is obvious that $F_{a} \cap F_{d}=\varnothing$ and that $F_{a} \cup F_{d}$ is linearly independent modulo $T(M(G))$. Notice that

$$
T\left(M_{a}(G)\right)^{-}=\operatorname{sp}\left(F_{a}\right)+T\left(M_{a}(G)\right) \subset \operatorname{sp}\left(F_{a}\right)+T(M(G)),
$$

and similarly for $M_{d}(G)$ and $F_{d}$.

Now suppose that $X$ is any two-sided invariant, closed subspace of $M(G)$ and that there exists a countable subset $F$ of $M(G)$ such that $T(X)^{-} \subset \operatorname{sp}(F)+T(M(G))$. We then claim that the unit ball $B_{1}(X)$ of $X$ is not weak-* dense in $B_{1}(M(G))$. Indeed, by a natural "two-sided" version of Lemma 2 , there exists a weak-* compact subset $W$ of $T(M(G))$ such that: if $G$ is noncompact, then $B_{1}(X) \subset W$; and if $G$ is compact, then $\mu-\lambda_{G} * \mu \in W$ for all $\mu \in B_{1}(X)$. First assume $G$ is noncompact. Since $W$ is weak-* compact, we then have $B_{1}(X)^{*} \subset W$, where $B_{1}(X)^{*}$ denotes the weak-* closure of $B_{1}(X)$ in $M(G)$. Since $W \subset T(M(G))$, we conclude that $\langle 1, \mu\rangle=0$ for all $\mu \in B_{1}(X)^{*}$; hence $B_{1}(X)$ is not weak-* dense in $B_{1}(M(G))$. Next assume that $G$ is compact (and infinite). Then $\mu-\lambda_{G} * \mu \in W$ for all $\mu \in B_{1}(X)^{*}$, again by the weak-* compactness of $W$. Taking discrete parts, we obtain $\mu_{d} \in(W)_{d} \subset$ $T\left(M_{d}(G)\right)$ for all $\mu \in B_{1}(X)^{*}$. A similar argument as above therefore shows that $B_{1}(X)$ is not weak-* dense in $B_{1}(M(G))$.

By the Hahn-Banach convexity theorem, both $B_{1}\left(M_{a}(G)\right)$ and $B_{1}\left(M_{d}(G)\right)$ are weak-* dense in $B_{1}(M(G))$. It follows from the above paragraph that neither $F_{a}$ nor $F_{d}$ is countable. In order to obtain uncountably many TILF's on $M(G)$ with the desired properties, it is sufficient to extend $\left\{\mu+T(M(G)): \mu \in F_{a} \cup F_{d}\right\}$ to a Hamel base of the quotient space $M(G) / T(M(G)$ ). (Notice here that the dimension of $T(M)^{-} / T(M)$ plays an important role.) This completes the proof. 
For two normed spaces $X$ and $Y$ on $G$, we define a norm on $X \cap Y$ by setting $\|f\|=\|f\|_{X}+\|f\|_{Y}$ for $f \in X \cap Y$. Notice that if both $X$ and $Y$ are Banach spaces on $G$, then so is $X \cap Y$.

Theorem $1^{*}$. Suppose $G$ is noncompact (and $\sigma$-compact). Let $X \subset L^{1}(G)$ be a left-invariant Fréchet space on $G$ whose topology is induced by countably many, left-invariant seminorms [e.g., $X=L^{1} \cap C_{0}$ ]. If $X$ contains an element with nonzero Haar integral, then there exist uncountably many TILF's on $L^{1}(G)$ whose restrictions to $X$ are linearly independent modulo $X^{\prime}$.

Proof. Unfortunately the closed unit ball of $L^{\prime}(G)$ is not $\sigma\left(C_{c}^{\prime}, C_{c}\right)$-compact, unless $G$ is discrete. So we replace $L^{1}(G)$ by $Y=M(G)$. Since the topology of $X$ is induced by left-invariant seminorms, each element of $X$ has bounded (left) orbit in $X$. Since $X \subset L^{1}(G) \subset M(G)$ and $G$ is noncompact, $X$ also satisfies condition $\left(\mathrm{C}_{3}\right)$ in Lemma 2.

Let $F$ be a subset of $T_{l}(X)^{-}$which is maximal with respect to the linear independence modulo $T_{l}(X)$. Let $F_{0}$ be a subset of $F$ which is maximal with respect to the linear independence modulo $T(M(G))$. Then we have

$$
T_{l}(X)^{-}=\operatorname{sp}(F)+T_{l}(X) \subset \operatorname{sp}\left(F_{0}\right)+T(M(G)) .
$$

Therefore the proofs of Lemmas 1 and 2 apply to the present situation mutatis mutandis. Since $X$ contains an element with nonzero Haar integral by the hypotheses, it follows from Lemma 2 that the set $F_{0}$ cannot be countable. This completes the proof.

REMARKS. (I) Let $G$ be an arbitrary LC group. If there exists a closed normal subgroup $H$ of $G$ such that $G / H$ is infinite and $\sigma$-compact, then $M(G)$ has uncountably many TILF's whose restrictions to $M_{a}(G)$ are linearly independent modulo $\left[M_{a}(G)\right]^{\prime}$. The proof is routine.

(II) It is possible to prove that if $G$ is a noncompact LCA group, then every translation invariant linear operator on $L^{1}(G)$ is continuous. (We omit the proof although it is nontrivial.) Thus the study of invariant functionals and the study of invariant operators are somewhat different (cf. [9]).

THEOREM 2. Suppose $1<p \leqslant \infty$. Then the following assertions are equivalent.

(i) Each LILF on $L^{p}(G)$ is either a constant multiple of the Haar integral (if $G$ is compact) or the zero functional (if $G$ is noncompact).

(ii) The dimension of the quotient space $L^{p}(G) / T_{l}\left(L^{p}(G)\right)$ is at most countable.

(iii) There exist a natural number $N$, a finite constant $C$, and a compact subset $K$ of $G$ such that each $f \in L^{p}(G)$ has a representation of the form

$$
f=a+\sum_{k=1}^{N}\left(f_{k}-\delta_{x_{k}} * f_{k}\right),
$$

where $a \in \mathbf{C}[a=0$ if $G$ is noncompact $], x_{k} \in K, f_{k} \in L^{p}(G)$, and $\left\|f_{k}\right\|_{p} \leqslant C\|f\|_{p}$ for all $k$ 's.

Proof. The space $X=Y=L^{p}(G)$ is a left-invariant Banach space on $G$ and possesses the three properties $\left(\mathrm{C}_{1}\right),\left(\mathrm{C}_{2}\right)$ and $\left(\mathrm{C}_{3}\right)$ in Lemma 2 . The only exceptional 
case arises when $G$ is noncompact and $p=\infty$, because then $L^{\infty}(G)$ does not satisfy $\left(\mathrm{C}_{3}\right)$. However, this difficulty may be circumvented as follows.

Suppose that $G$ is noncompact (and $\sigma$-compact) and that $T_{l}\left(C_{0}(G)\right)^{-} \subset \operatorname{sp}(F)+$ $T_{l}\left(L^{\infty}(G)\right)$ for some countable subset $F$ of $L^{\infty}(G)$. It is obvious that $X=C_{0}(G)$ satisfies conditions $\left(\mathrm{C}_{2}\right)$ and $\left(\mathrm{C}_{3}\right)$ in Lemma 2 . It follows from Lemma 2 with $Y=L^{\infty}(G)$ that there exists a natural number $N$ and a compact subset $K$ of $G$ such that $B_{1}\left(C_{0}(G)\right)$ is contained in the weak-* compact subset $W_{N, K}$ of $T_{l}\left(L^{\infty}(G)\right)$. But then $B_{1}\left(L^{\infty}(G)\right) \subset W_{N, K}$, since $B_{1}\left(C_{0}(G)\right)$ is weak-* dense in $B_{1}\left(L^{\infty}(G)\right)$. We have thus proved that (ii) $\Rightarrow$ (iii) holds even in the exceptional case under discussion. This completes the proof.

THEOREM 2*. Let $G$ be a $\sigma$-compact, noncompact, amenable LC group, and let $1<p \leqslant \infty$. Then there exist uncountably many LILF's on $L^{1}(G)+L^{p}(G)$ whose restrictions to $L^{p} \cap C_{0}(G)$ are linearly independent modulo the continuous linear functionals on $L^{p} \cap C_{0}(G)$.

Proof. The space $Y=M(G)+L^{p}(G)$ forms a Banach space on $G$ with respect to the intermediate norm defined by

$$
\|f\|=\inf \left\{\|g\|_{M}+\|h\|_{p}: g \in M(G), h \in L^{p}(G), \& g+h=f\right\} .
$$

It is easy to check that $X=L^{p} \cap C_{0}(G)$ and $Y$ satisfy conditions $\left(\mathrm{C}_{1}\right)-\left(\mathrm{C}_{3}\right)$ in Lemma 2.

Suppose, by way of contradiction, that $T_{l}(X)^{-} \subset \operatorname{sp}(F)+T_{l}(Y)$ for some countable subset $F$ of $Y$. Then Lemma 2 yields a $\sigma\left(Y, C_{c}\right)$-compact subset $W_{N, K}$ of $T_{l}(Y)$ such that $B_{1}\left(L^{p} \cap C_{0}\right) \subset W_{N, K}$. By using an appropriate approximate identity, one checks that $B_{1}\left(L^{p} \cap C_{0}\right)$ is $\sigma\left(C_{c}^{\prime}, C_{c}\right)$-dense in $B_{1}\left(L^{p} \cap L^{\infty}\right)$; hence $B_{1}\left(L^{p} \cap L^{\infty}\right) \subset$ $W_{N, K}$. The remainder of the proof may be accomplished by modifying Woodward's method in [22, Theorem 1], as follows:

Since $B_{1}\left(L^{p} \cap L^{\infty}\right) \subset W_{N, K}$, each element $f$ of $L^{p} \cap L^{\infty}$ can be written in the form

$$
f=\mu+\sum_{k=1}^{N}\left(h_{k}-\delta_{x_{k}} * h_{k}\right),
$$

where $\mu \in M(G), h_{k} \in L^{p}(G)$ and $x_{k} \in K$ for all $k$ 's. Since $G$ is assumed to be amenable, it satisfies the Følner condition [5]. We can, therefore, find a sequence $\left(E_{n}\right)$ of compact subsets of $G$ such that

$$
\lambda_{G}\left(E_{n} \Delta x^{-1} E_{n}\right)<n^{-1} \lambda_{G}\left(E_{n}\right) \quad(x \in K, n \in \mathbf{N}),
$$

where $\Delta$ stands for symmetric difference of sets. There is no loss of generality in assuming that $\lambda_{G}\left(E_{n}\right)>n^{2}$ for all $n \geqslant 1$. Writing $q=p /(p-1)$, we infer from (9) and Hölder's inequality that $h \in L^{p}(G)$ and $x \in K$ imply

$$
\begin{aligned}
\left|\int_{E_{n}}\left(h-\delta_{x} * h\right) d x\right| & \leqslant \int_{E_{n} \Delta x^{-1} E_{n}}|h| d x \leqslant \lambda_{G}\left(E_{n} \Delta x^{-1} E_{n}\right)^{1 / q}\|h\|_{p} \\
& \leqslant n^{-1 / q} \lambda_{G}\left(E_{n}\right)^{1 / q}\|h\|_{p}
\end{aligned}
$$


for all $n \geqslant 1$. It follows from (8) that $f \in L^{p} \cap L^{\infty}$ implies

$$
\begin{aligned}
\left|\int_{E_{n}} f d x\right| & \leqslant\|\mu\|+n^{-1 / q} \lambda_{G}\left(E_{n}\right)^{1 / q} \sum_{k=1}^{N}\left\|h_{k}\right\|_{p} \\
& \leqslant C_{f} \cdot n^{-1 / q} \lambda_{G}\left(E_{n}\right)^{1 / q} \quad(n=1,2, \ldots),
\end{aligned}
$$

where $C_{f}$ is a finite constant depending only on $f$. Therefore the Banach-Steinhaus theorem yields a finite constant $C$ such that

$$
\left|\int_{E_{n}} f d x\right| \leqslant C n^{-1 / q} \lambda_{G}\left(E_{n}\right)^{1 / q}\left(\|f\|_{p}+\|f\|_{\infty}\right)
$$

for all $f \in L^{p} \cap L^{\infty}$ and all $n$ 's. In particular, choosing $f$ to be the indicator function of $E_{n}$, we obtain

$$
\begin{aligned}
\lambda_{G}\left(E_{n}\right) & \leqslant C n^{-1 / q} \lambda_{G}\left(E_{n}\right)^{1 / q}\left[\lambda_{G}\left(E_{n}\right)^{1 / p}+1\right] \\
& \leqslant 2 C n^{-1 / q} \lambda_{G}\left(E_{n}\right) \quad(n=1,2, \ldots) .
\end{aligned}
$$

Since $q$ is a finite positive number, the last inequalities give us the desired contradiction.

THEOREM 3. Let $X$ be a left-invariant closed subspace of $L^{\infty}(G)$ such that $B_{1}(X)$ is weak-* dense in $B_{1}\left(L^{\infty}\right)$ [e.g., $C_{0}(G), C_{u}(G), C(G)$, etc.]. Suppose either:

(i) $G$ is compact and $L^{\infty}(G)$ has two linearly independent $L I L F$ 's,

(ii) $G$ is noncompact and $L^{\infty}(G)$ has a nonzero $L I L F$, or

(iii) $l^{\infty}(G)$ has a LILF which does not annihilate $C(G)$.

Then there exist uncountably many LILF's on $L^{\infty}(G)$ whose restrictions to $X$ are linearly independent modulo $X^{\prime}$.

Proof. A moment's glance at the proof of Theorem 2 shows that the desired conclusion is certainly true if either (i) or (ii) holds. So it will suffice to show that (iii) implies (i) if $G$ is compact, and (ii) if $G$ is noncompact. If $G$ is discrete, then there is nothing to prove. So assume $G$ is nondiscrete.

Passing to a nondiscrete metrizable quotient of $G$ (cf. [7, p. 71]), we can find a compact subset $E$ of $G$ such that $\lambda_{G}(E)>0$ and $E$ has empty interior. Then no finitely many translates of $E$ cover $G$ (a.e.) by the Baire category theorem. Since the maximal ideal space of $L^{\infty}(G)$ is compact, it follows that there exists a nonzero complex homomorphism $\Phi$ of $L^{\infty}(G)$ such that $\Phi\left(\delta_{x} * \xi_{E}\right)=0$ for all $x \in G$. $\left(\xi_{E}\right.$ denotes the indicator function of $E$.) It is easy to see that such a $\Phi$ can be chosen so that $\Phi(f)=f(e)$ for all $f \in C(G)$, where $e$ is the identity element of $G$. Define a mapping $\Phi^{\prime}$ from $L^{\infty}(G)$ into $l^{\infty}(G)$ by setting

$$
\left(\Phi^{\prime} f\right)(x)=\Phi\left(\delta_{x^{-1}} * f\right) \quad\left(f \in L^{\infty}(G) \text { and } x \in G\right) .
$$

Thus $\Phi^{\prime} \xi_{E}=0$ and $\left(\Phi^{\prime} f\right)(x)=f(x)$ for all $f \in C(G)$. Moreover, $f \in L^{\infty}(G), a \in G$, and $x \in G$ imply

$$
\Phi^{\prime}\left(\delta_{a} * f\right)(x)=\Phi\left(\delta_{x^{-1}} * \delta_{a} * f\right)=\left(\Phi^{\prime} f\right)\left(a^{-1} x\right)
$$

by (11). It follows immediately that $\Phi^{\prime}\left[T_{l}\left(L^{\infty}(G)\right)\right] \subset T_{l}\left(l^{\infty}(G)\right)$. 
Now suppose that either (i) or (ii) fails to hold. Then $L^{\infty}(G)=\operatorname{sp}\left(\xi_{E}\right)+T_{l}\left(L^{\infty}(G)\right)$ by Theorem 2 with $p=\infty$. Since $C(G) \subset L^{\infty}(G)$ and $\Phi^{\prime} \xi_{E}=0$, it follows that $C(G)=\Phi^{\prime}[C(G)] \subset T_{l}\left(l^{\infty}(G)\right)$. This contradicts (iii) and the proof is complete.

Corollary 4. Let $G$ be a $\sigma$-compact, infinite, LC group. If $G$ is amenable as a discrete group, a fortiori, if $G$ is abelian, then there exist uncountably many LILF's on $L^{\infty}(G)$ whose restrictions to $C_{0}(G)$ are linearly independent modulo $\left[C_{0}(G)\right]^{\prime}$.

Proof. Obvious from the definition of amenability.

REMARKS. (III) Whenever $G$ is a nondiscrete $\sigma$-compact LC group, there exist uncountably many TILF's on $M(G)+L^{\infty}(G)$ whose restrictions to $L^{1}(G)$ are linearly independent modulo $\left[L^{1}(G)\right]^{\prime}$. This may be proved by modifying the proof of Theorem 1 .

(IV) $L^{\infty}(G)$ has a discontinuous LILF if and only if so does $C_{l u}(G)$, the Banach space of all left uniformly continuous bounded functions on $G$. The "only if" part is a special case of Theorem 3. The proof of the "if" part requires Cohen's factorization theorem [2]; see also [6].

(V) Let $\operatorname{LIM}\left(L^{\infty}(G)\right)$ denote the set of all left-invariant means on $L^{\infty}(G)$. As is well known, if $G$ is an infinite LC group which is amenable as a discrete group, then the dimension of [the linear space spanned by] $\operatorname{LIM}\left(L^{\infty}(G)\right)$ is quite "huge"; see $[\mathbf{1}, 3-5,18-21]$. This can also happen for some nonamenable groups.

Suppose that $G$ and $H$ are two infinite compact groups, and that $G$ is amenable as a discrete group. For each $M \in \operatorname{LIM}\left(L^{\infty}(G)\right)$, define

$$
\left\langle f, M \times \lambda_{H}\right\rangle=\left\langle\int f(x, y) d \lambda_{H}(y), M_{x}\right\rangle \quad\left(f \in L^{\infty}(G \times H)\right) .
$$

It is obvious that this is well defined and that the correspondence $M \rightarrow M \times \lambda_{H}$ is an isomorphism of $\operatorname{LIM}\left(L^{\infty}(G)\right)$ into $\operatorname{LIM}\left(L^{\infty}(G \times H)\right)$. We do not know whether condition (iii) in Theorem 3 characterizes the amenable groups.

(VI) Let $G$ be a free group with two generators (cf. [7]). It is a well-known fact that $l^{\infty}(G)$ has no left-invariant mean. In fact, the zero functional is the only one LILF on $l^{\infty}(G)$. Therefore the conclusion of Theorem 3 fails to hold for some groups.

Let $a$ and $b$ be the free generators of $G$, and let $f \in l^{\infty}(G)$ be given. We claim that $f$ has a representation of the form

$$
f(x)=g(a x)-g(x)+h(b x)-h(x) \quad(x \in G)
$$

for some $g$ and $h \in l^{\infty}(G)$. To confirm this, notice that $G$ is the union of the sets $\{e\}$, $A$ and $B$, where $A[B]$ is the set of all reduced words starting with a nonzero power of $a[b]$. Therefore we can write $f=f_{1}+f_{2}$, where $f_{1}, f_{2} \in l^{\infty}(G), \operatorname{supp} f_{1} \subset A \cup\{e\}$, and $\operatorname{supp} f_{2} \subset B \cup\{e\}$. Using the fact that $G$ is the union of the disjoint sets $b^{n}(A \cup\{e\})$ for $n=0, \pm 1, \ldots$, we define

$$
h\left(b^{n} x\right)= \begin{cases}f_{1}(x) & \text { if } x \in A \cup\{e\} \text { and } n>0 \\ 0 & \text { if } x \in A \cup\{e\} \text { and } n \leqslant 0\end{cases}
$$


Similarly, define

$$
g\left(a^{n} x\right)= \begin{cases}f_{2}(x) & \text { if } x \in B \cup\{e\} \text { and } n>0 \\ 0 & \text { if } x \in B \cup\{e\} \text { and } n \leqslant 0\end{cases}
$$

One checks that $f_{1}(x)=h(b x)-h(x)$ and $f_{2}(x)=g(a x)-g(x)$ for all $x \in G$. Hence $f=f_{1}+f_{2}$ admits a decomposition of the desired form. It follows immediately that the only LILF on $l^{\infty}(G)$ is the trivial functional.

(VII) Let $G$ be an arbitrary LC group. Suppose $G$ contains a closed normal subgroup $H$ such that $G / H$ is compact and $C(G / H)$ admits a discontinuous LILF. Then there exist uncountably many LILF's on $C_{c}(G)$ which are linearly independent modulo $C_{c}^{\prime}(G)$. This may be proved by using Theorem (15.21) of [7] and applying Lemma 2 (cf. [17]).

EXAMpLEs. (a) Let $A_{1}(\mathbf{R})$ denote the space of all $f \in L^{1} \cap C^{\infty}(\mathbf{R})$ such that $\operatorname{supp} \hat{f} \subset[-1,1]$ and $f^{(n)} \in L^{1}(\mathbf{R})$ for all $n \in \mathbf{N}$. Here $\hat{f}$ denotes the Fourier transform of $f$. It is evident that $A_{1}(\mathbf{R})$ forms an invariant Fréchet space on $\mathbf{R}$ with respect to the seminorms

$$
p_{n}(f)=\|f\|_{1}+\left\|f^{\prime}\right\|_{1}+\cdots+\left\|f^{(n)}\right\|_{1} \quad(n=1,2, \ldots) .
$$

Let $Y$ denote the linear span in $C_{c}^{\prime}(\mathbf{R})$ of all $M(\mathbf{R})+L^{p}(G)$ with $1 \leqslant p<\infty$. Then $Y$ has uncountably many TILF's whose restrictions to $A_{1}(\mathbf{R})$ are linearly independent modulo $\left[A_{1}(\mathbf{R})\right]^{\prime}$.

To prove this, put $Y_{m}=M(\mathbf{R})+L^{m}(\mathbf{R})$ for $m \geqslant 1$. Then $Y_{m} \subset Y_{m+1}$ and $Y$ is the union of all $Y_{m}$ with $m \in \mathbf{N}$. Accordingly, $Y$ may be regarded as the strict inductive limit of the invariant Banach spaces $Y_{m}(m \in \mathbf{N})$. Suppose by way of contradiction that the above-stated result is false. Then we can modify the proofs of Lemmas 1 and 2 to obtain three natural numbers $N, q$ and $n$ with the following property: each $f \in A_{1}(\mathbf{R})$ can be written in the form

$$
f=\sum_{j=1}^{N}\left(\mu_{j}-\delta_{x_{j}} * \mu_{j}\right)+\sum_{j=1}^{N}\left(h_{j}-\delta_{x_{j}} * h_{j}\right),
$$

where $\mu_{j} \in M(\mathbf{R}), h_{j} \in L^{q}(\mathbf{R}), x_{j} \in[-N, N],\left\|\mu_{j}\right\|_{M} \leqslant N p_{n}(f)$, and $\left\|h_{j}\right\|_{q} \leqslant N p_{n}(f)$ for all $j$ 's.

Now put $I_{m}=[-m, m]$ for $m=1,2, \ldots$ Then notice that the symmetric difference $\left\{[-N, N]+I_{m}\right\} \Delta I_{m}$ has Lebesgue measure $2 N$ and is disjoint from $I_{m}$. If $f \in A_{1}(\mathbf{R})$ is as above, we infer from Hölder's inequality that

$$
\begin{aligned}
\left|\int_{-m}^{m} f d x\right| & \leqslant \sum_{j=1}^{N}\left|\mu\left(\left[I_{m}-x_{j}\right] \Delta I_{m}\right)\right|+\sum_{j=1}^{N} \int_{\left[I_{m}-x_{j}\right] \Delta I_{m}}\left|h_{j}\right| d x \\
& \leqslant o(1)+\sum_{j=1}^{N}\left\{\int_{\left[I_{m}-x_{j}\right] \Delta I_{m}}\left|h_{j}\right|^{q} d x\right\}^{1 / q}(2 N)^{(q-1) / q} \\
& =o(1)+o(1)=o(1) \text { as } m \rightarrow \infty .
\end{aligned}
$$

Therefore, $\int_{\mathbf{R}} f d x=0$ for all $f \in A_{1}(\mathbf{R})$, which is of course absurd. 
(b) As was proved by Meisters [10], each TILF on $C^{\infty}(\Pi)$ is continuous and is therefore a constant multiple of the Lebesgue integral. However, this is not true for any $C^{(m)}(\Pi)=\left\{f \in C(\Pi): f^{(m)} \in C(\Pi)\right\}$, where $m \in \mathbf{N}$.

To prove this, fix $m \in \mathbf{N}$ and let $Y_{m}$ denote the subspace of all $f \in C^{(m-1)}(\Pi)$ such that $f^{(m-1)}$ is absolutely continuous and $f^{(m)} \in L^{\infty}(\Pi)$. Then $Y_{m}$ forms an invariant Banach space on $\Pi$ with respect to the norm

$$
\|f\|_{(m)}=\|f\|_{\infty}+\left\|f^{\prime}\right\|_{\infty}+\cdots+\left\|f^{(m)}\right\|_{\infty} \quad\left(f \in Y_{m}\right) .
$$

One checks that the imbedding of $C^{(m)}(\Pi)$ into $Y_{m}$ is isometric, that $B_{1}\left(Y_{m}\right)$ is $\sigma\left(Y_{m}, C(\Pi)\right)$-compact, and that the unit ball of $C^{(m)}(\Pi)$ is $\sigma\left(Y_{m}, C(\Pi)\right)$-dense in $B_{1}\left(Y_{m}\right)$. Suppose that $X=C^{(m)}(\Pi)$ has no discontinuous TILF. Then Lemmas 1 and 2 assure that each $f \in Y_{m}$ can be written in the form

$$
f=\langle 1, f\rangle+\sum_{j=1}^{N}\left(h_{j}-\delta_{x_{j}} * h_{j}\right),
$$

where $h_{j} \in Y_{m}$ and $x_{j} \in \Pi$ for all $j$ 's. Taking the $m$ th derivatives of both sides of the last equation, we obtain

$$
f^{(m)}=\sum_{j=1}^{N}\left[h_{j}^{(m)}-\delta_{x_{j}} * h_{j}^{(m)}\right] .
$$

However, it is easy to see that $(d / d t)^{m} Y_{m}$ consists of all $g \in L^{\infty}(\Pi)$ with $\langle 1, g\rangle=0$. It follows from Theorem 3 that the last expression is impossible for some $f \in Y_{m}$. This reductio ad absurdum establishes the desired result. Notice also that each TILF on $C^{(m)}(\Pi)$ which extends to a TILF on $L^{2}(\Pi)$ is necessarily continuous by [15].

(c) The following example is included here because of its contrast with the last example. Let $G$ be a compact abelian group with dual $\Gamma$, and let $\left(\psi_{n}\right)$ be a sequence of elements of $l^{\infty}(\Gamma)$ such that $1 \leqslant \psi_{1} \leqslant \psi_{2} \leqslant \cdots$. Let $X$ consist of all $f \in C(G)$ such that

$$
p_{n}(f)=\sum\left\{|\hat{f}(\gamma)| \cdot \psi_{n}(\gamma): \gamma \in \Gamma\right\}<\infty \quad(n=1,2, \ldots) .
$$

It is obvious that $X$ forms an invariant Fréchet space on $G$ with respect to the seminorms $p_{n}(\cdot)$. Suppose that there exists a compact subgroup $H$ of $G$ such that $G / H$ is an infinite torsion group. Then it is easy to construct an element $f_{0} \in X$ with the following property: whenever $F$ is a finite subset of $G$, then $\lambda_{H(F)} * f_{0}$ is nonconstant, where $H(F)$ is the compact subgroup of $G$ generated by $H \cup F$ and $\lambda_{H(F)}$ is the normalized Haar measure of $H(F)$.

Suppose by way of contradiction that there is no TILF on $L^{1}(G)$ whose restriction to $X$ is discontinuous. Then, by Lemmas 1,2 and the Lebesgue-Radon-Nikodým theorem, we can write $f_{0}$ in the form

$$
f_{0}=C+\sum_{j=1}^{N}\left(g_{j}-\delta_{x_{j}} * g_{j}\right),
$$

where $C=\left\langle 1, f_{0}\right\rangle, g_{j} \in L^{1}(G)$ and $x_{j} \in G$ for all $j$ 's. Letting $F=\left\{x_{1}, \ldots, x_{N}\right\}$, we then have $\lambda_{H(F)} * f_{0}=C$ a.e. on $G$. Since $f_{0}$ is continuous, it follows that $\lambda_{H(F)} * f_{0}$ $=C$ everywhere on $G$, which contradicts our choice of $f_{0}$. 
I would like to thank Professor R. B. Burckel for providing me with useful references.

\section{REFERENCES}

1. C. Chou, The exact cardinality of the set of invariant means on a group, Proc. Amer. Math. Soc. 55 (1976), 103-106.

2. P. J. Cohen, Factorization in group algebras, Duke Math. J. 26 (1959), 199-205.

3. E. Granirer, On left amenable semigroups which admit countable left-invariant means, Bull. Amer. Math. Soc. 69 (1963), 101-105.

4. . On the invariant mean on topological semigroups and on topological groups, Pacific J. Math. 15 (1965), 107-140.

5. F. P. Greenleaf, Invariant means on topological groups and their applications, Math. Studies, no 16, Van Nostrand, New York, 1969.

6. E. Hewitt, The ranges of certain convolution operators, Math. Scand. 15 (1964), 147-155.

7. E. Hewitt and K. A. Ross, Abstract harmonic analysis, Vol. I, Springer-Verlag, Berlin, Gottingen and Heidelberg, 1963

8. M. Jerison and W. Rudin, Translation-invariant functionals, Proc. Amer. Math. Soc. 13 (1962), $417-423$

9. C. J. Lester, Continuity of operators on $L^{2}(G)$ and $L^{1}(G)$ commuting with translations, J. London Math. Soc. 11 (1975), 144-146.

10. G. H. Meisters, Translation-invariant linear forms and a formula for the Dirac measure, J. Funct. Anal. 8 (1971), 173-188.

11. __ Some discontinuous translation-invariant linear forms, J. Funct. Anal. 12 (1973), 199-210.

12. A Guichard theorem on connected monothetic groups, Studia Math. 43 (1973), 161-163.

13. __ Periodic distributions and non-Liouville numbers, J. Funct. Anal. 26 (1977), 68-88.

14. Some problems and results on translation-invariant linear forms, Preprint, 1982.

15. G. H. Meisters and W. M. Schmidt, Translation-invariant linear forms on $L^{2}(G)$ for compact abelian groups, J. Funct. Anal. 11 (1972), 407-424.

16. A. P. Robertson and W. J. Robertson, Topological vector spaces, Cambridge Univ. Press, London and New York, 1973.

17. W. Roelcke, L. Asam, S. Dierolf and P. Dierolf, Discontinuous translation-invariant linear forms on $K(G)$, Math. Ann. 239 (1979), 219-222.

18. J. M. Rosenblatt, Invariant means for the bounded meosurable functions on a non-discrete locally compact group, Math. Ann. 220 (1976), 219-228.

19. The number of extensions of an invariant mean. Compositio Math. 33 (1976), 147-159.

20. J. M. Rosenblatt and M. Talagrand, Different types of invariant means, J. London Math. Soc. 24 (1981), 525-532.

21. W. Rudin, Invariant means on $L^{\infty}$, Studia Math. 44 (1972), 219-227.

22. G. S. Woodward, Translation-invariant linear forms on $C_{0}(G), C(G), L^{p}(G)$ for noncompact groups, J. Funct. Anal. 16 (1974), 205-220.

Department of Mathematics, Kansas State University, Manhattan, Kansas 66506 